\title{
A highly accurate and efficient analytical approach to bridge deck free vibration analysis
}

\author{
D.J. Gorman ${ }^{\mathrm{a}, *}$ and L. Garibaldi ${ }^{\mathrm{b}}$ \\ ${ }^{a}$ Department of Mechanical Engineering, University \\ of Ottawa, 770 King Edward Avenue, Ottawa, Canada \\ ${ }^{\mathrm{b}}$ Dipartimento di Meccanico, Politecnico di Torin, \\ Torino, Italy
}

Received 16 May 2000

Revised 12 September 2000

The superposition method is employed to obtain an accurate analytical type solution for the free vibration frequencies and mode shapes of multi-span bridge decks. Free edge conditions are imposed on the long edges running in the direction of the deck. Inter-span support is of the simple (knife-edge) type. The analysis is valid regardless of the number of spans or their individual lengths. Exact agreement is found when computed results are compared with known eigenvalues for bridge decks with all spans of equal length. Mode shapes and eigenvalues are presented for typical bridge decks of three and four span lengths. In each case torsional and non-torsional modes are studied.

\section{Introduction}

At the Dipartimento di Meccanica of the Polytecnico di Torino, of Turin Italy, an extended study of the dynamic response of bridge decks to vehicular excitation is in progress. The study involves the taking of vibration measurements on actual bridges and development of theoretical models. The long term goal is to develop accurate modeling of bridge dynamic behavior in response to the above excitation. Ultimately, knowledge gained thereby will be utilized to achieve optimization in bridge design.

\footnotetext{
* Corresponding author: D. J. Gorman, Dep't. Mech. Eng., Univ. of Ottawa, 770 King Edward Ave. K1N 6N5, Ottawa, Canada. Email: dgorman@genie.uottawa.ca.
}

As a first step it was desired to develop analytical means for accurately predicting free vibration frequencies and mode shapes of the bridge decks. The bridge response to forced excitation would then be represented in terms of these mode shapes.

It was recognized that in a multi-span deck many closely spaced frequencies (eigenvalues) must be anticipated and a careful delineation must be made between adjacent eigenvalues and their associated mode shapes. For these reasons it was concluded that a highly accurate analytical type analysis based on continuum mechanics was essential. Furthermore, results obtained in this manner could augment, and help verify, results of numerical studies carried out by a finite element method.

Such an analysis, based on the superposition method, is described in this paper for the first time. It will be seen that the governing differential equation is satisfied exactly throughout the structure. Boundary conditions are satisfied to any desired degree of accuracy. Convergence is rapid and the method is found to be highly efficient from a computational point of view.

While there is a massive literature available related to single span plate vibration (the classical rectangular plate) and a limited literature related to double span plates there does not appear to be publications of the type discussed here dealing with vibration of plates of any arbitrary number of spans.

\section{Mathematical procedure}

\subsection{Development of building block solutions}

For illustrative purposes we examine the behavior of a three-span deck. It will be obvious to the reader that the same analytical technique is easily extended to handle decks of any number of spans.

The three-span deck utilized for illustrative purposes is represented schematically in Fig. 1. Each span has 


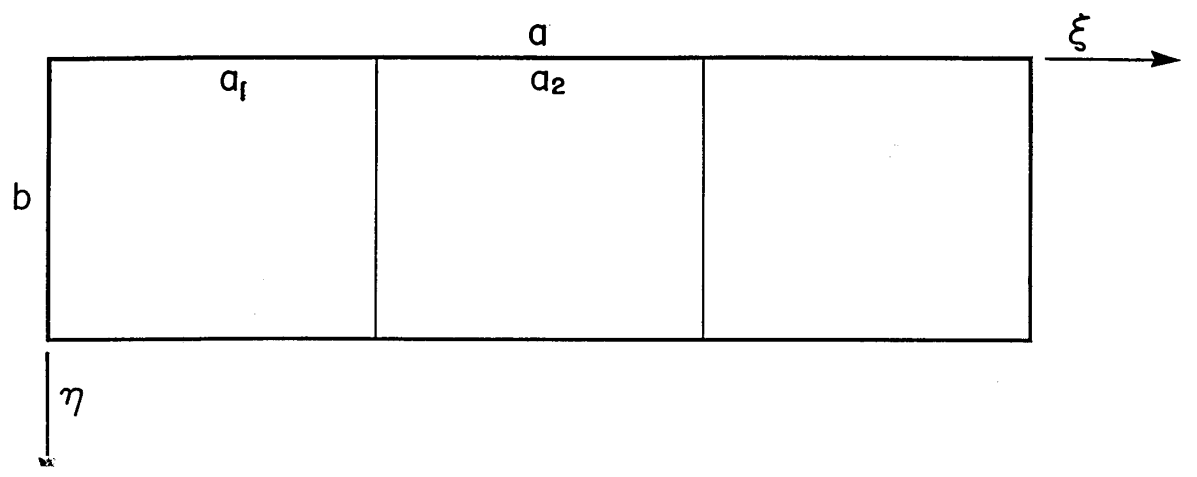

Fig. 1. Schematic representation of 3-span bridge deck.

a common width ' $b$ '. The first and second spans have lengths $a_{1}$, and $a_{2}$, respectively. Edges along the deck entrance and exit are given simple support. Simple line support is provided along the interface of the spans. We may think of this latter support as being of the knifeedge type. All edges running in the direction of the long axis of the deck are considered to be free.

A solution for the free vibration frequencies and mode shapes of the deck as described above is obtained by superimposing a set of rectangular plate forced vibration problems, called building blocks, and constraining constants appearing in these solutions so that prescribed boundary conditions are satisfied by the superimposed set. The building blocks to be superimposed here are shown schematically in Fig. 2.

Each building block has a rectangular area coinciding with the entire area of the bridge deck. Overall length of the deck is denoted by the symbol ' $a$ '. The first building block has simple support conditions imposed along the edges $\xi=0$, and $\xi=1$. Slip-shear conditions are imposed along the edge, $\eta=0$. This condition is denoted by two small circles adjacent to the edge. It implies that vertical edge reaction and slope taken normal to the edge are everywhere zero.

The edge, $\eta=1$, is also free of vertical edge reaction but it is driven by a distributed harmonic edge rotation of circular frequency $\omega$.Our first task is to determine the response of this building block to the harmonic excitation.

We represent the distribution of amplitude of the imposed rotation as,

$$
\left.\frac{\partial W(\xi, \eta)}{\partial \eta}\right|_{\eta=1}=\sum_{m=1,2}^{\infty} E_{m} \sin m \pi \xi
$$

It will be obvious that the amplitude of the response of the building block can be expressed in the form proposed by Levy as,

$$
W(\xi, \eta)=\sum_{m=1,2}^{\infty} Y_{m}(\eta) \sin m \xi
$$

Substituting equation 2 into the well known differential equation governing the behaviour of thin plates it is found that variables are separable and we obtain two possible forms of solution for the functions $Y_{m}(\eta)$, They are [1],

$$
\begin{aligned}
& \text { For } \lambda^{2}>(m \pi)^{2} \\
& \qquad \begin{aligned}
Y_{m}(\eta)= & A_{m} \sin h \beta_{m} \eta+B_{m} \cos h \beta_{m} \eta \\
& +C_{m} \sin \gamma_{m} \eta+D_{m} \cos \gamma_{m} \eta
\end{aligned}
\end{aligned}
$$

and, For $\lambda^{2}<(m \pi)^{2}$,

$$
\begin{aligned}
Y_{m}(\eta)= & A_{m} \sin h \beta_{m} \eta+B_{m} \cos h \beta_{m} \eta \\
& +C_{m} \sin h \gamma_{m} \eta+D_{m} \cos h \gamma_{m} \eta
\end{aligned}
$$

Here $A_{m}$ and $B_{m}$, etc., are constants to be evaluated while, $\beta_{m}^{2}=\phi^{2}\left(\lambda^{2}+(m \pi)^{2}\right)$, and $\gamma_{m}^{2}=$ $\phi^{2}\left(\lambda^{2}-(m \pi)^{2}\right)$ or $\phi^{2}\left((m \pi)^{2}-\lambda^{2}\right)$ whichever is positive.

It will be obvious that due to symmetry about the $\xi$ axis, the constants $A_{m}$, and $C_{m}$, above must equal zero.

There remain two other constants to be determined and two boundary conditions to be satisfied. They become, for any value of $m$,

$$
\frac{\partial^{3} W(\xi, \eta)}{\partial \eta^{3}}+\left.\nu^{*} \frac{\partial^{3} W(\xi, \eta)}{\partial \eta \partial \xi^{2}}\right|_{\eta=1}=0
$$

and

$$
\left.Y_{m}^{\prime}(\eta)\right|_{\eta=1}=E_{m}
$$

Equation 5 expresses the condition of zero vertical edge reaction along the driven edge. Equation 6 follows from equations 1 and 2. Substituting equation 2 into equation 5 and enforcing boundary conditions represented by equations 5 and 6 , one obtains, 


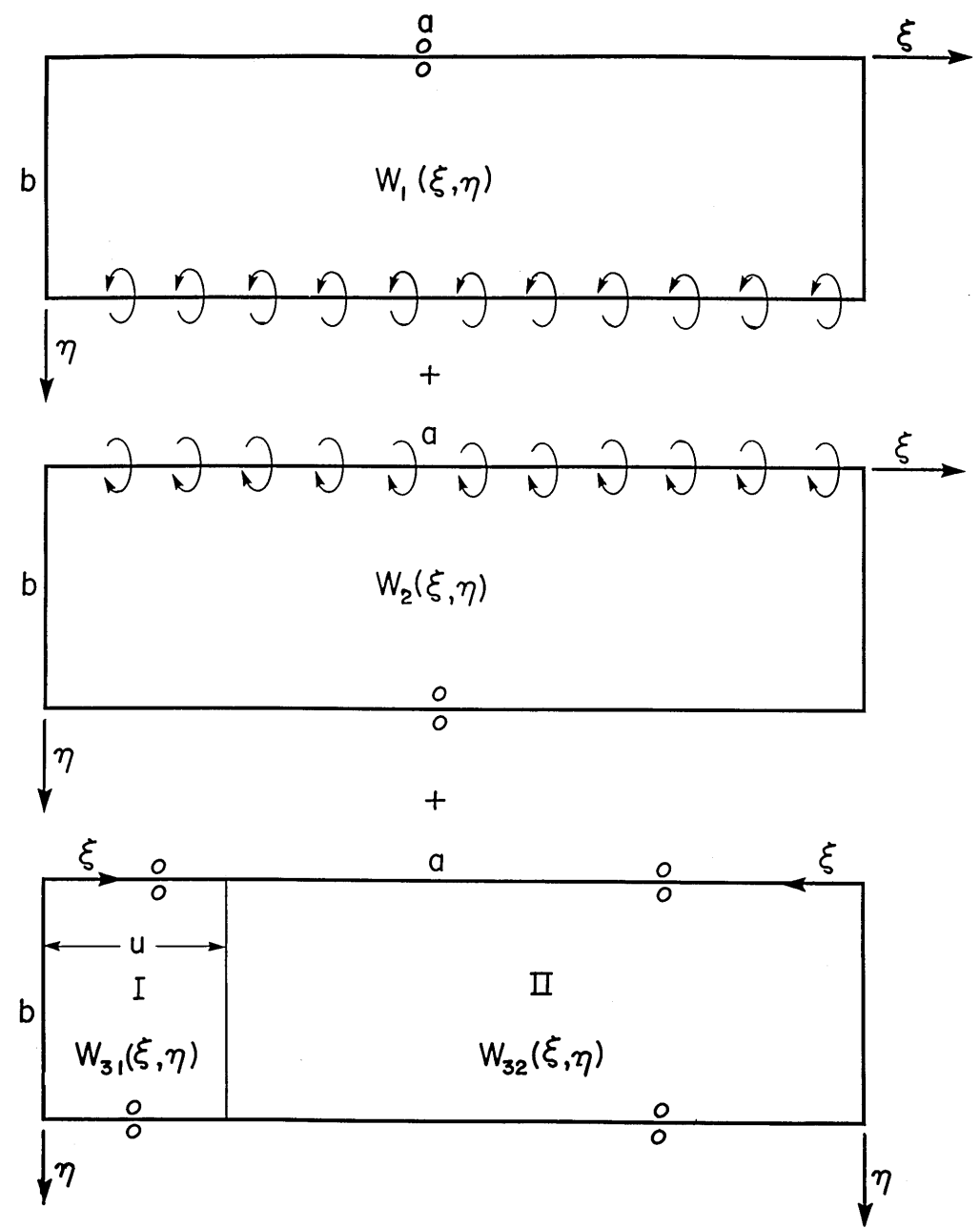

Fig. 2. Schematic representation of the three building blocks employed in solving the bridge deck free vibration problem.

$$
\text { For } \begin{aligned}
& \lambda^{2}>(m \pi)^{2}, \\
& Y_{m}(\eta) \\
= & E_{m} \theta_{11 m}\left\{\cos h \beta_{m} \eta+\theta_{1 m} \cos \gamma_{m} \eta\right\}
\end{aligned}
$$

where,

$$
=\frac{-\beta_{m}\left(\beta_{m}^{2}-\nu^{*} \phi^{2}(m \pi)^{2}\right) \sin h \beta_{m}}{\gamma_{m}\left(\gamma_{m}^{2}+\nu^{*} \phi^{2}(m \pi)^{2}\right) \sin \gamma_{m}}
$$

and $\theta_{11 m}=1 /\left(\beta_{m} \sin h \beta_{m}-\theta_{1 m} \gamma_{m} \sin \gamma_{m}\right)$ and, for $\lambda^{2}<(m \pi)^{2}$,

$$
\begin{aligned}
Y_{m}(\eta)= & E_{m} \theta_{22 m} \\
& \left\{\cos h \beta_{m} \eta+\theta_{2 m} \cos h \gamma_{m} \eta\right\}
\end{aligned}
$$

where,

$$
\theta_{2 m}=\frac{-\beta_{m}\left(\beta_{m}^{2}-\nu^{*} \phi^{2}(m \pi)^{2}\right) \sin h \beta_{m}}{\gamma_{m}\left(\gamma_{m}^{2}-\nu^{*} \phi^{2}(m \pi)^{2}\right) \sin h \gamma_{m}}
$$
and $\theta_{22 m}=1 /\left(\beta_{m} \sin h \beta_{m}+\theta_{2 m} \gamma_{m} \sin \gamma_{m}\right)$

We therefore have the exact response of the first building block to any imposed harmonic driving slope with amplitude as represented by equation 1 .

The second building block of Fig. 2 differs from the first only in that it is driven by an imposed harmonic slope along the edge, $\eta=0$. It will be obvious that solution for response of this building block can be obtained from that of the first by simply replacing the quantity $\eta$ of equations 7 and 8 with the quantity $1-\eta$. In view of sign conventions one should also precede this latter solution with a negative sign.

Finally, we turn to the third building block of Fig. 2 . It is given slip-shear support along the edges, $\eta=0$, and $\eta=1$. Simple support is imposed along the other 


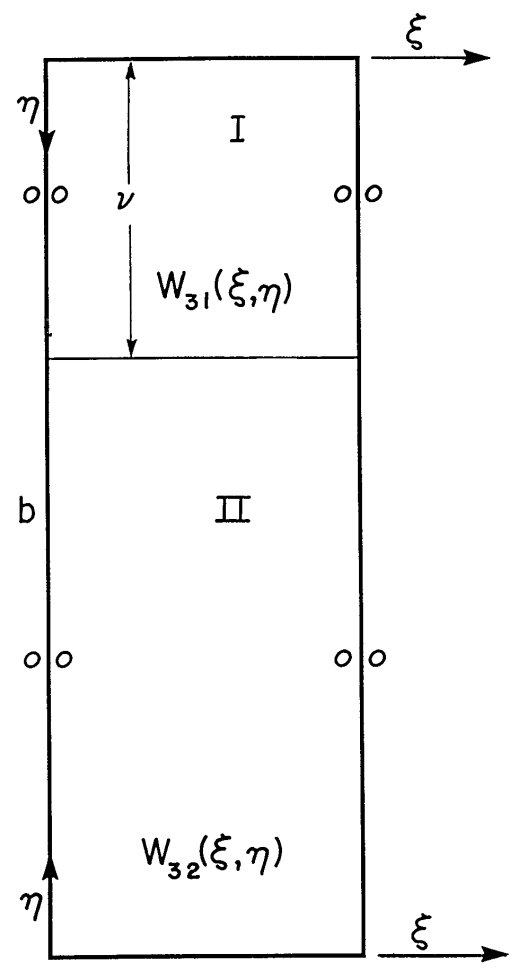

Fig. 3. Schematic representation of intermediate building block utilized in order to arrive at solution for third building block of Fig. 2.

edges. This building block is driven by a harmonic force distributed along a line dimensionless distance ' $u$ ' from the $\eta$ axis. The distributed amplitude of this driving force will be expressed in series form.

In order to arrive at a solution for response of the third building block it is found advantageous to begin by analyzing the building block represented in Fig. 3 . It is seen that this latter is essentially a mirror image of the former. We denote its upper and lower segments by the Roman numerals I and II, respectively. A solution for the upper segment is expressed as,

$$
W_{31}(\xi, \eta)=\sum_{m=0,1}^{\infty} Y_{m}(\eta) \cos m \pi \xi
$$

where $\xi$, and $\eta$, are coordinates attached to the segment. A solution in identical form is used to represent the response of the lower segment.

Fortunately, it is found that a solution for this building block response is essentially available at the beginning of the eight chapter of Reference [2]. There are only two minor differences. First, the edges $\xi=0$, and $\xi=1$, of the earlier solution were given simple support. Therefore sine functions were used instead of the cosine functions of equation 9. Secondly, a con- centrated force was applied in the earlier solution. This force was represented by a sine series expansion of the Dirac function. Here we will use a general cosine series as no Dirac function is required. Guided by the earlier solution we express the amplitude of the distributed harmonic line force in dimensionless form as,

$$
P^{*}(\xi)=\sum_{m=0,1}^{\infty} E_{m s}(m) \cos m \pi \xi
$$

Solutions for the displacements $W_{31}(\xi, \eta)$ and $W_{32}(\xi, \eta)$ related to the segments of the present problem are obtained following exactly the same steps as described in the earlier reference. In fact, our solution can be extracted directly from the earlier one.

For any value of ' $m$ ' solutions for the functions $Y_{m}(\eta)$ will take a form as given by equations 3 or 4 . There will obviously be eight unknowns to be determined. Four of these are eliminated because of antisymmetry required about the boundaries $\eta=0$. A set of four equations relating the other four unknowns is obtained through enforcing continuity of displacement, slope, and bending moment across the interface of the segments. Also, the difference in shear force across this boundary must balance the applied driving force.

Fortunately, it is shown in reference [2] that an exact solution is easily obtained for this set of four simultaneous algebraic equations. Solution for response of the building block of Fig. 3 is extracted from that provided for the problem of the reference discussed above, as follows, for $\lambda^{2}>(m \pi)^{2}$, for segment I

$$
Y_{m}(\eta)=A_{m} \sin h \beta_{m} \eta+B_{m} \sin \gamma_{m} \eta
$$

for segment II

$$
Y_{m}(\eta)=C_{m} \sin h \beta_{m} \eta+D_{m} \sin \gamma_{m} \eta
$$

with,

$$
\begin{aligned}
A_{m} & =\frac{E_{m s}(m) \sin h\left(\beta_{m}(1-\nu)\right)}{\left(\beta_{m}^{2}+\gamma_{m}^{2}\right) \beta_{m} \sin h \beta_{m}} \\
B_{m} & =\frac{-E_{m s}(m) \sin \left(\gamma_{m}(1-\nu)\right)}{\left(\beta_{m}^{2}+\gamma_{m}^{2}\right) \gamma_{m} \sin \gamma_{m}} \\
C_{m} & =\frac{E_{m s}(m) \sin h\left(\beta_{m} \nu\right)}{\left(\beta_{m}^{2}+\gamma_{m}^{2}\right) \beta_{m} \sin h \beta_{m}} \\
D_{m} & =\frac{-E_{m s}(m) \sin \left(\gamma_{m} \nu\right)}{\left(\beta_{m}^{2}+\gamma_{m}^{2}\right) \gamma_{m} \sin \gamma_{m}}
\end{aligned}
$$

and, for $\lambda^{2}<(m \pi)^{2}$, for segment I,

$$
Y_{m}(\eta)=A_{m} \sin h \beta_{m} \eta+B_{m} \sin h \gamma_{m} \eta
$$

for segment II 


$$
Y_{m}(\eta)=C_{m} \sin h \beta_{m} \eta+D_{m} \sin h \gamma_{m} \eta
$$

with,

$$
\begin{aligned}
& A_{m}=\frac{E_{m s}(m) \sin h\left(\beta_{m}(1-\nu)\right)}{\left(\beta_{m}^{2}-\gamma_{m}^{2}\right) \beta_{m} \sin h \beta_{m}} \\
& B_{m}=\frac{-E_{m s}(m) \sin h\left(\gamma_{m}(1-\nu)\right)}{\left(\beta_{m}^{2}-\gamma_{m}^{2}\right) \gamma_{m} \sin h \gamma_{m}} \\
& C_{m}=\frac{E_{m s}(m) \sin h\left(\beta_{m} \nu\right)}{\left(\beta_{m}^{2}-\gamma_{m}^{2}\right) \beta_{m} \sin h \beta_{m}} \\
& D_{m}=\frac{-E_{m s}(m) \sin h\left(\gamma_{m} \nu\right)}{\left(\beta_{m}^{2}-\gamma_{m}^{2}\right) \gamma_{m} \sin h \gamma_{m}}
\end{aligned}
$$

$\beta_{m}^{2}$ and $\gamma_{m}^{2}$ are as defined earlier in this paper. We therefore have available the exact response of the building block of Fig. 3 for any harmonic line force excitation. It will be noted that the above quantities $A_{m}$, $B_{m}$, etc., differ from those of equations 8.21 and 8.22 of reference [2] only in that the coefficients $E_{m s}(m)$ replace the quantity $P^{*} \sin (m \pi u)$ utilized earlier.

Finally, a solution for the response of the third building block of Fig. 2 is extracted from that of Fig. 3 through a simple interchange of axis. We will utilize the sub-script ' $n$ ' in connection with this building block in order to avoid confusion with solutions for the first two.

Response for segments I and II are written in series form as,

$$
W_{31}(\xi, \eta)=\sum_{n=0,1}^{\infty} Y_{n 1}(\xi) \cos n \pi \eta
$$

and

$$
W_{32}(\xi, \eta)=\sum_{n=0,1}^{\infty} Y_{n 2}(\xi) \cos n \pi \eta
$$

In order to infer the solution for this problem from that of Fig. 3 through an interchange of axis certain rules must be followed [1]. The quantity $\lambda^{2}$ must be replaced by $\lambda^{2} \phi^{2}$ and the aspect ratio $\phi$ appearing in the previous solution must be replaced by its inverse. We also replace dimensionless quantity ' $v$ ' with ' $u$ ', and in view of the definition of $P^{*}$ we should divide the new coefficients by $\phi^{4}$. We could alternatively, consider the latter quantity to be absorbed in the driving coefficients $E_{n s}(n)$.

It follows that the quantities $\beta_{n}^{2}$ and $\gamma_{n}^{2}$ become,

$$
\begin{aligned}
& \beta_{n}^{2}=\left\{\lambda^{2} \phi^{2}+(n \pi)^{2}\right\} / \phi^{2} \text { and } \\
& \gamma_{n}^{2}=\left\{\lambda^{2} \phi^{2}-(n \pi)^{2}\right\} / \phi^{2} \text { or } \\
& \left\{(n \pi)^{2}-\lambda^{2} \phi^{2}\right\} / \phi^{2}
\end{aligned}
$$

whichever is positive.

We then have, for $\lambda^{2} \phi^{2}>(n \pi)^{2}$

$$
Y_{n 1}(\xi)=A_{n} \sin h \beta_{n} \xi+B_{n} \sin \gamma_{n} \xi
$$

and

$$
Y_{n 2}(\xi)=C_{n} \sin h \beta_{n} \xi+D_{n} \sin \gamma_{n} \xi
$$

where,

$$
\begin{aligned}
& A_{n}=\frac{E_{n s}(n) \sin h\left(\beta_{n}(1-u)\right)}{\phi^{4}\left(\beta_{n}^{2}+\gamma_{n}^{2}\right) \beta_{n} \sin h \beta_{n}} \\
& B_{n}=\frac{-E_{n s}(n) \sin \left(\gamma_{n}(1-u)\right)}{\phi^{4}\left(\beta_{n}^{2}+\gamma_{n}^{2}\right) \gamma_{n} \sin \gamma_{n}} \\
& C_{n}=\frac{E_{n s}(n) \sin h\left(\beta_{n} u\right)}{\phi^{4}\left(\beta_{n}^{2}+\gamma_{n}^{2}\right) \beta_{n} \sin h \beta_{n}} \\
& D_{n}=\frac{-E_{n s}(n) \sin \left(\gamma_{n} u\right)}{\phi^{4}\left(\beta_{n}^{2}+\gamma_{n}^{2}\right) \gamma_{n} \sin \gamma_{n}}
\end{aligned}
$$

while for $\lambda^{2} \phi^{2}<(n \pi)^{2}$

$$
Y_{n 1}(\xi)=A_{n} \sin h \beta_{n} \xi+B_{n} \sin h \gamma_{n} \xi
$$

and

$$
Y_{n 2}(\xi)=C_{n} \sin h \beta_{n} \xi+D_{n} \sin h \gamma_{n} \xi
$$

where,

$$
\begin{aligned}
A_{n} & =\frac{E_{n s}(n) \sin h\left(\beta_{n}(1-u)\right)}{\phi^{4}\left(\beta_{n}^{2}-\gamma_{n}^{2}\right) \beta_{n} \sin h \beta_{n}} \\
B_{n} & =\frac{-E_{n s}(n) \sin h\left(\gamma_{n}(1-u)\right)}{\phi^{4}\left(\beta_{n}^{2}-\gamma_{n}^{2}\right) \gamma_{n} \sin h \gamma_{n}} \\
C_{n} & =\frac{E_{n s}(n) \sin h\left(\beta_{n} u\right)}{\phi^{4}\left(\beta_{n}^{2}-\gamma_{n}^{2}\right) \beta_{n} \sin h \beta_{n}} \\
D_{n} & =\frac{-E_{n s}(n) \sin h\left(\gamma_{n} u\right)}{\phi^{4}\left(\beta_{n}^{2}-\gamma_{n}^{2}\right) \gamma_{n} \sin h \gamma_{n}}
\end{aligned}
$$

We thus have available the response of the third building block of Fig. 2 to any harmonic line force excitation.

\section{Development of eigenvalue matrix}

Generation of the eigenvalue matrix turns out to be a fairly simple and straightforward exercise. Regardless of the number of spans in the bridge deck only the basic building blocks of Fig. 2 are required. The first two are always required. One building block, equivalent to the third of Fig.2, is required to take care of each of the inter-span line supports. Two will be required, of course, for analysis of the illustrative three-span deck. 


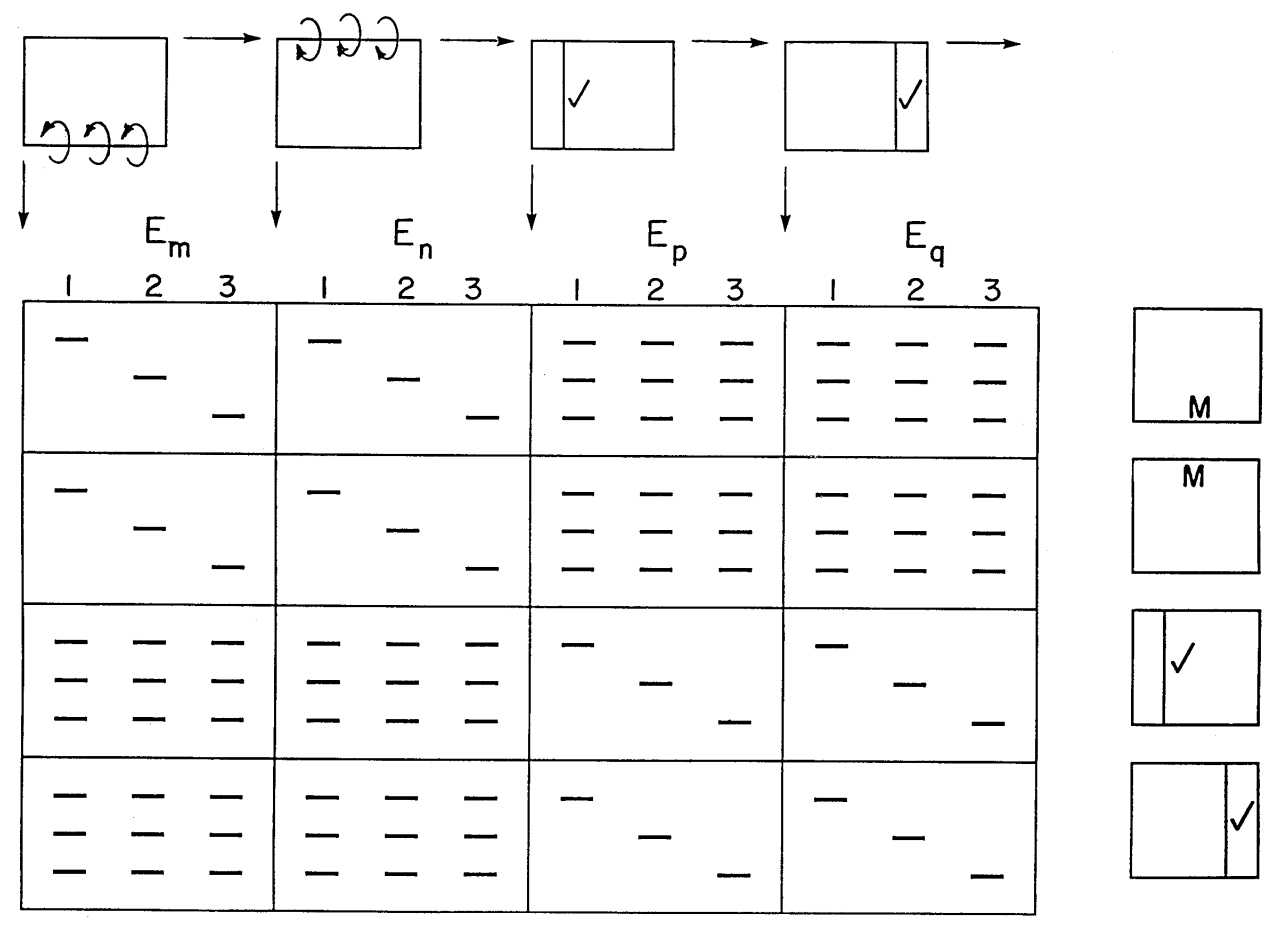

Fig. 4. Schematic representation of the eigenvalue matrix required for analyzing 3-span brudge deck.

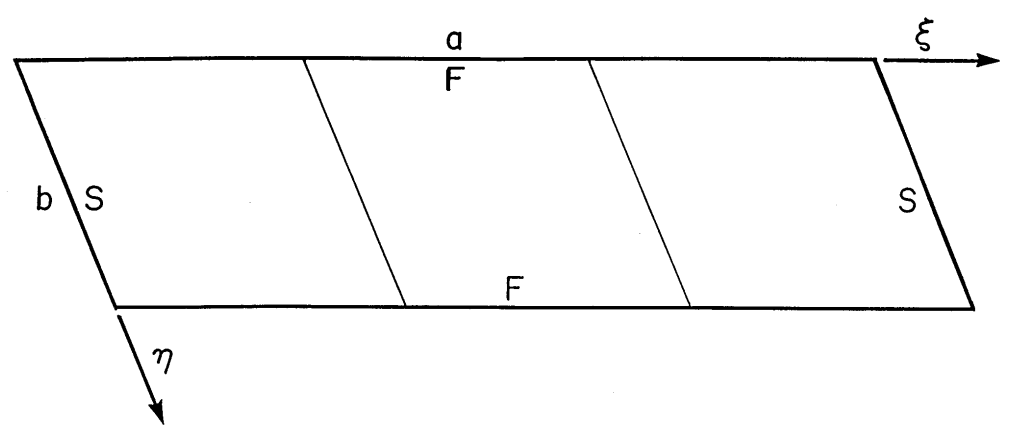

Fig. 5. Rectangular plate with opposite edges simply supported, and free. Transvers node lines are those of higher mode vibration.

The first will be characterized by a dimensionless distance, $u_{1}=a_{1} / a$, between the $\eta$ axis and the location of the harmonic line loading. For the second, the dimensionless distance will be, $u_{2}=\left(a_{1}+a_{2}\right) / a$.

Let all of the building blocks be driven by $\mathrm{K}$ Fourier driving components. We begin by superimposing the four building blocks, one-upon-the-other, and expanding their contributions toward bending moment along the edge, $\eta=1$, in a sine series of $\mathrm{K}$ terms. By arbitrarily utilizing a sine series we take advantage of orthogonality. To fulfill the required condition of zero net bending moment we set each net coefficient in this new series equal to zero. This gives rise to a set of $\mathrm{K}$ homogeneous algebraic equations relating the $4 \mathrm{~K}$ unknown driving coefficients. Another set is obtained in an identical manner by enforcing the condition of zero net bending moment along the edge, $\eta=0$.

Finally, two additional sets, each of K equations, are obtained by expanding the net displacement along each of the span interfaces in a cosine series and setting each of the net coefficients equal to zero. Here the cosine series permits taking advantage of orthogonality.

The eigenvalue matrix associated with a 3-span bridge deck is depicted schematically in Fig. 4, based on three term expansions. Small inset figures above the matrix indicate the building blocks involved and 


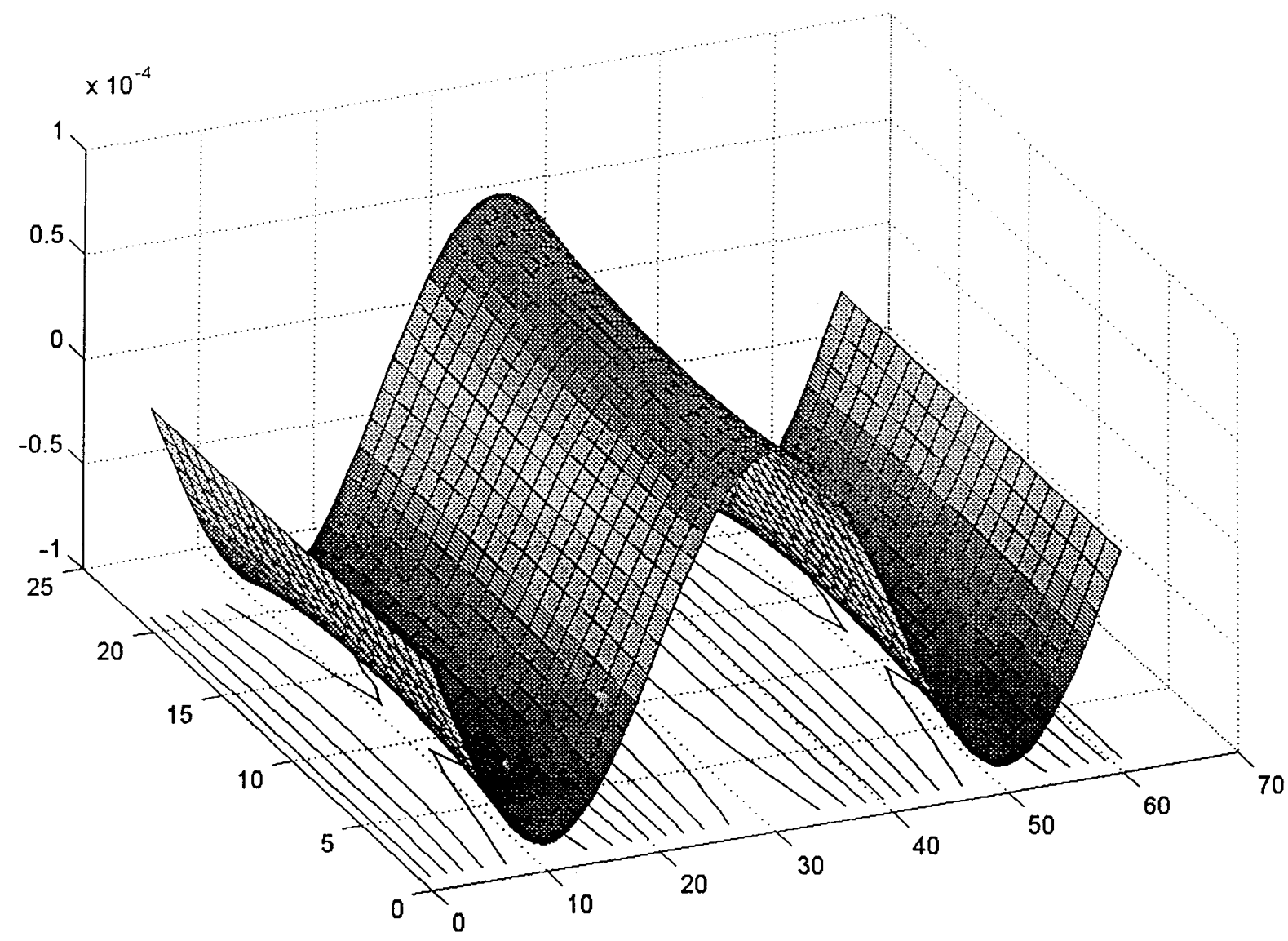

Fig. 6. First mode shape of 3-span deck, $\phi_{1}=\phi_{3}=1.0, \phi_{2}=1.1$.

those to the right indicate the boundary conditions to be satisfied. It will be noted that off-diagonal elements in various segments of the matrix are zero.

\section{Presentation of computed results}

Eigenvalues are obtained following established procedures. One searches for those values of $\lambda^{2}$ which cause the determinant of the eigenvalue matrix to vanish (the eigenvalues). Having established an eigenvalue, one sets one of the non-zero driving coefficients equal to unity and solves the resulting set of non-homogeneous algebraic equations to obtain values for the remaining driving coefficients. The mode shape associated with the eigenvalue can then be generated. It is necessary to chooze a sufficiently high value for the parameter $\mathrm{K}$, the number of terms in the solution series, in order to obtain the desired level of convergence.

In order to select an appropriate value for $\mathrm{K}$ it is desirable to conduct convergence tests. The results of a
Table 1

Computed first mode eigenvalue, $\lambda^{2}$ vs. $K$, number of terms in solution series, for 3-span deck. $\phi_{1}=\phi_{3}=1.0, \phi_{2}=1.25$.

\begin{tabular}{rcrccccc}
\hline$K$ & $\lambda^{2}$ & $K$ & $\lambda^{2}$ & $K$ & $\lambda^{2}$ & $K$ & $\lambda^{2}$ \\
\hline 3 & 10.649 & 7 & 10.650 & 11 & 10.651 & 15 & 10.651 \\
4 & 10.649 & 8 & 10.650 & 12 & 10.651 & 16 & 10.651 \\
5 & 10.650 & 9 & 10.651 & 13 & 10.651 & 17 & 10.651 \\
6 & 10.650 & 10 & 10.651 & 14 & 10.651 & 18 & 10.651 \\
\hline
\end{tabular}

typical convergence test are tabulated in Table 1 . This test is related to computation of the first mode eigenvalue for a three-span deck with $\phi_{1}=\phi_{3}=1.0$, and $\phi_{2}=1.25$. Before conducting the test we must decide upon how many significant digits we wish to have in the computed eigenvalues. Here, we are interested in four significant digits. We therefore tabulate computed eigenvalues to five significant digits so that rate of convergence can be better studied.

The reader will appreciate that in order to have at least one half-sine wave available to represent the lateral deflection of each span we must utilize a value of $\mathrm{K}$ which is equal or greater than three. Furthermore, as K 


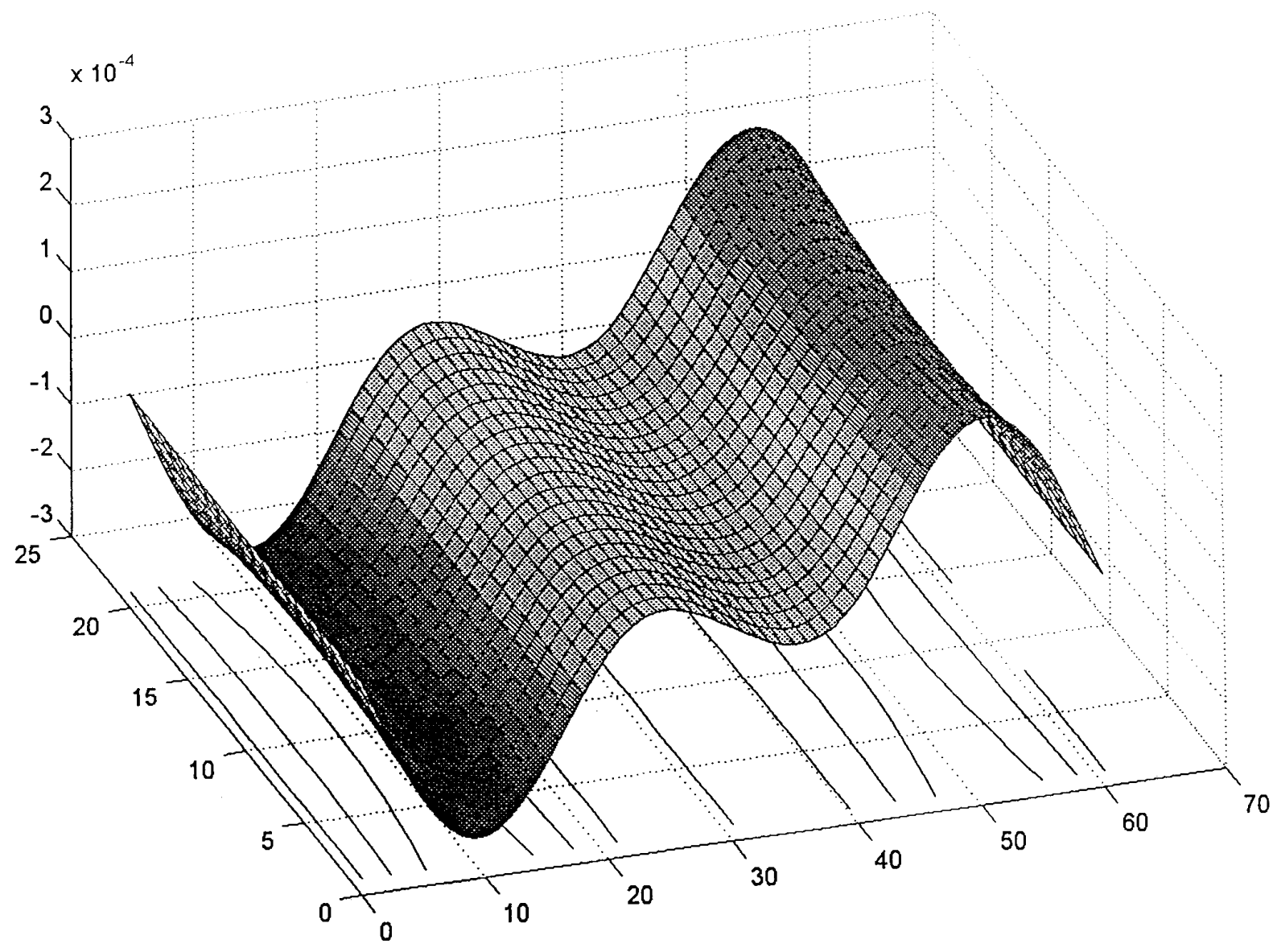

Fig. 7. Second mode shape of 3-span deck, $\phi_{1}=\phi_{3}=1.0, \phi_{2}=1.1$.

takes on a value of six we expect to have approximately two half-sine waves available for each span. With $\mathrm{K}$ equal to nine, approximately three half-sine waves per span will be available, etc.

Examining Table 1 it is seen that only the fifth digit in computed eigenvalues changes (by 0.001) as we increase $\mathrm{K}$ from three to six. Increasing $\mathrm{K}$ from nine to twelve results in only a further increase of 0.001 , i.e., a minimal increment in the fifth digit. Beyond a value of $\mathrm{K}$ equal nine, up to a value of eighteen, there is no change, even in the fifth digit. It is obvious that this represents a high rate of convergence. Even with a minimum possible value of $K$, i.e., $K$ equals three, we have four digit accuracy giving an eigenvalue of 10.65 . This high rate of convergence, and hence high degree of accuracy in the computed eigenvalues, is typical of that encountered with use of the superposition method.

It will be obvious that for bridge decks with all spans of equal length there are certain eigenvalues which can be predicted in advance. Essentially exact solutions are available in the literature for free vibration of plates with two opposite simply supported edges, the other two being free [2]. Consider a rectangular plate of this type, of the same geometry as the bridge deck, with simple support along the edges $\xi=0$ and $\xi=1$. Such a plate is shown schematically in Fig. 5. In some of its higher modes it may have transverse running node lines which coincide with the inter-span support lines of the bridge deck of interest. We expect these mode shapes and frequencies to coincide with certain of the computed mode shapes and frequencies of the actual deck. In such cases we say that the bridge deck supports are inactive. These predicted exact frequencies, obtained from classical plate theory, serve as a valuable check on our analysis.

An alternative but much more demanding free vibration analysis of the general multi-span bridge deck may be achieved by selecting appropriate sets of building blocks for each span, individually, and then enforcing inter-span continuity conditions. Such an analysis has 


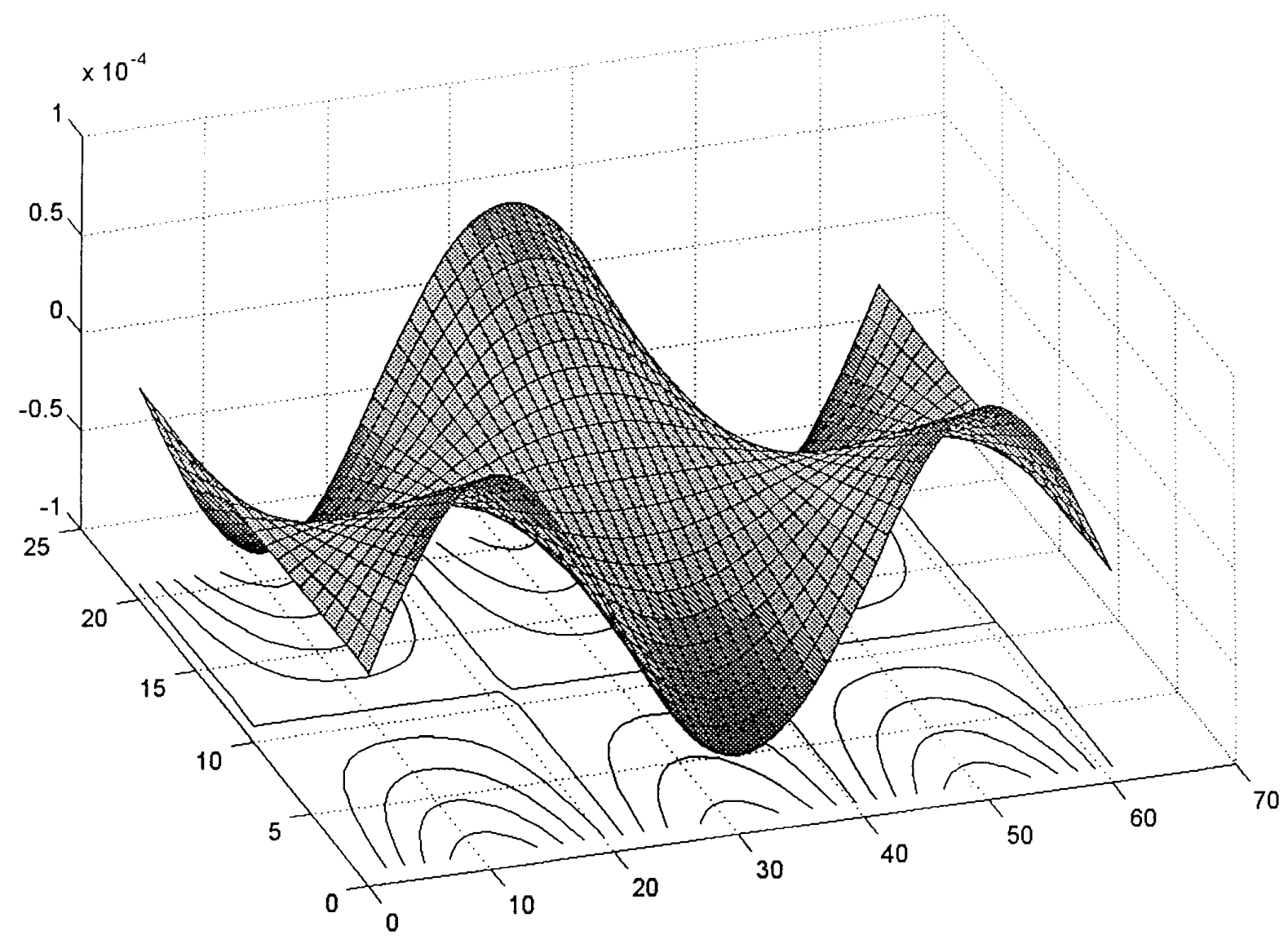

Fig. 8. Third mode shape of 3-span deck, $\phi_{1}=\phi_{2}=1.0, \phi_{2}=1.1$.

in fact been performed, although it has not been published at this time. Nevertheless, this 'multi-span' approach has been utilized to verify eigenvalues obtained by the 'line loading' continuous plate method which has been described in detail here. Results obtained by the present method are presented in the tables to follow, along with verification results obtained by the 'multi-span' approach.

The reader will appreciate that only a very limited amount of computed results can be presented. Accordingly it has been chosen to first present computed eigenvalues for a three-span deck with aspect ratios of the first and third spans set at 1.0. Aspect ratio of the center span is allowed to vary from 1.0 to 1.25 . It has been found that certain free vibration modes for decks with all spans of aspect ratio 1.0, coincide with the classical, or 'inactive support', modes discussed earlier. Eigenvalues associated with these modes, and computed here, are found to agree exactly. They are indicated in the tables by means of an asterisk.
Computations related to results presented here were carried out with a value of K equal to 19 .

In Table 2 computed eigenvalues are presented for the first six modes of a three-span deck for various values of the center span aspect ratio. It will be noted, as expected, that eigenvalues increase as the parameter $\phi_{2}$ increases. This is because the center span is decreasing and the deck is becoming stiffer. We note also, for example, that eigenvalues for the fourth and fifth modes, with $\phi_{2}=1.0$, are very close together. This is to be anticipated with multi-span decks, as stated earlier, and the analyst is thus cautioned to make careful delineation between the various deck natural frequencies and mode shapes when conducting such a study.

It will also be noted that there is extremely good agreement between the eigenvalues computed here and those computed by the auxiliary 'multi-span approach', for the purposes of comparison. It is pointed out that while both are essentially superposition methods, they involve vastly different approaches to the same problem. The present line-loading method is computation- 


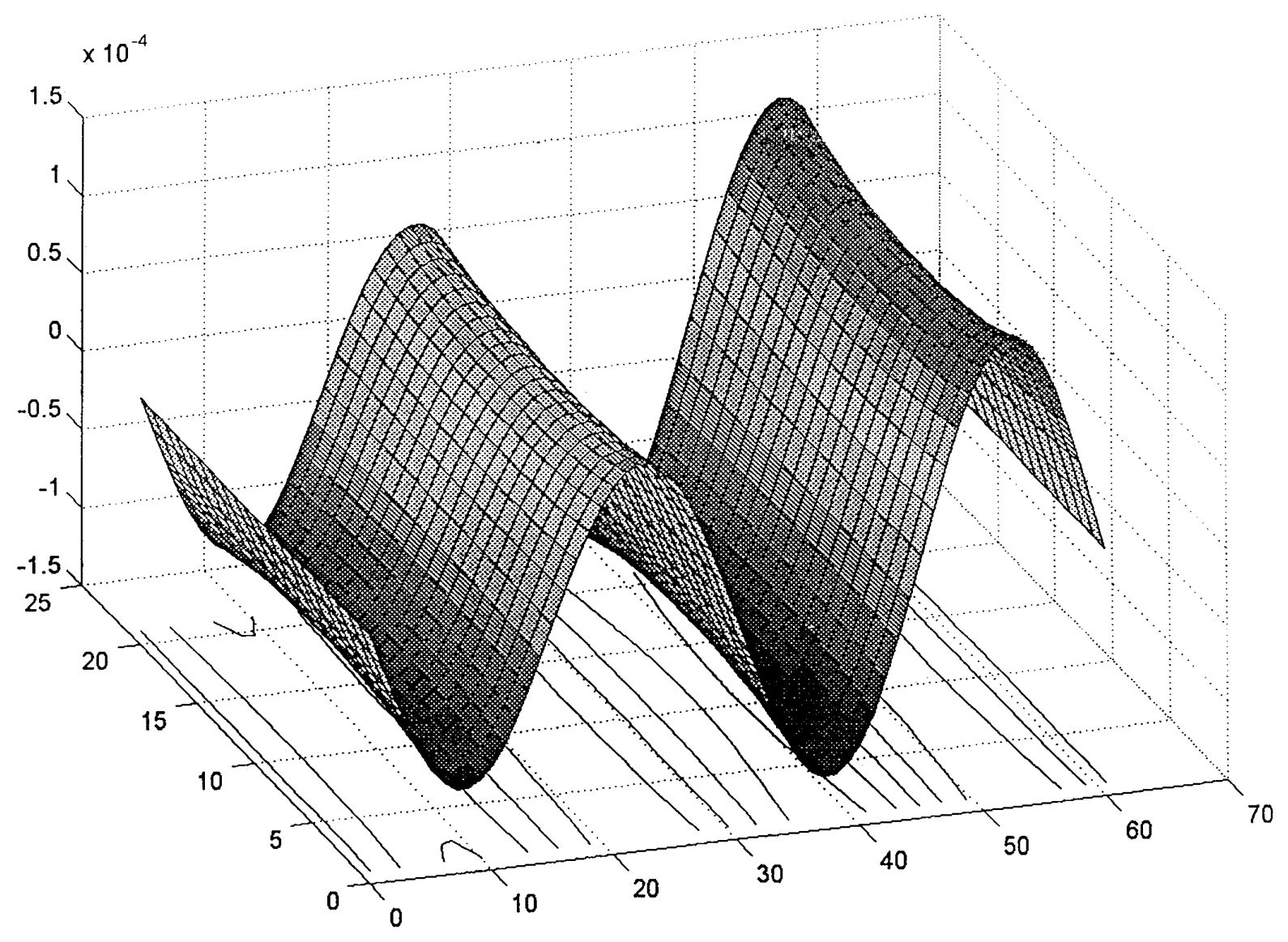

Fig. 9. First mode shape of 4-span deck, $\phi_{1}=\phi_{3}=\phi_{4}=1.0, \phi_{2}=1.1$.

Table 2

Computed 3 - Span Deck Eigenvalues, $\lambda_{b}^{2}=\omega b^{2} \sqrt{ } \rho / D, \phi_{1}=\phi_{3}=1.0$. Comparison values obtained from Multi-Span Approach, in brackets (* indicates classical values)

\begin{tabular}{cllllllll}
\hline Mode & \multicolumn{8}{c}{$\phi_{2}$} \\
\cline { 2 - 8 } & \multicolumn{9}{c}{1.0} & \multicolumn{7}{c}{1.15} & \multicolumn{2}{c}{1.25} \\
\hline 1 & $9.568^{*}$ & $(9.568)$ & 9.864 & $(9.864)$ & 10.32 & $(10.32)$ & 10.65 & $(10.65)$ \\
2 & 12.36 & $(12.36)$ & 12.44 & $(12.44)$ & 12.59 & $(12.59)$ & 12.72 & $(12.72)$ \\
3 & $15.88^{*}$ & $(15.88)$ & 16.20 & $(16.20)$ & 16.63 & $(16.63)$ & 16.90 & $(16.90)$ \\
4 & 18.04 & $(18.04)$ & 18.10 & $(18.11)$ & 18.18 & $(18.19)$ & 18.28 & $(18.27)$ \\
5 & 18.17 & $(18.18)$ & 19.29 & $(19.30)$ & 21.74 & $(21.75)$ & 24.39 & $(24.38)$ \\
6 & 22.93 & $(22.96)$ & 24.19 & $(24.16)$ & 26.91 & $(26.94)$ & 29.94 & $(29.91)$ \\
\hline
\end{tabular}

ally much more efficient. It is found in Table 2 that the two sets of results are identical to four significant digits for the first three modes. Very slight variations in the fourth significant digit are observed in some of the higher modes. These observations justify a very high degree of confidence in the analytical method described here as well as the tabulated results.

In part to demonstrate the versatility of this computational method, eigenvalues for a 4-span deck are tabulated in Table 3. Here, aspect ratios of the first, third, and fourth span are fixed at 1.0, while that of the second span is allowed to vary from 1.0 to 1.25 . Almost the same comments made with regard to Table 2 can be made with regard to this Table. Again, eigenvalues increase as the deck becomes stiffer. Again, there is extremely good agreement between results obtained by means of the two different computational methods. It will be appreciated that the eigenvalue matrix for this problem will simply be an augmented matrix of the kind shown in Fig. 4. It will be augmented with one 


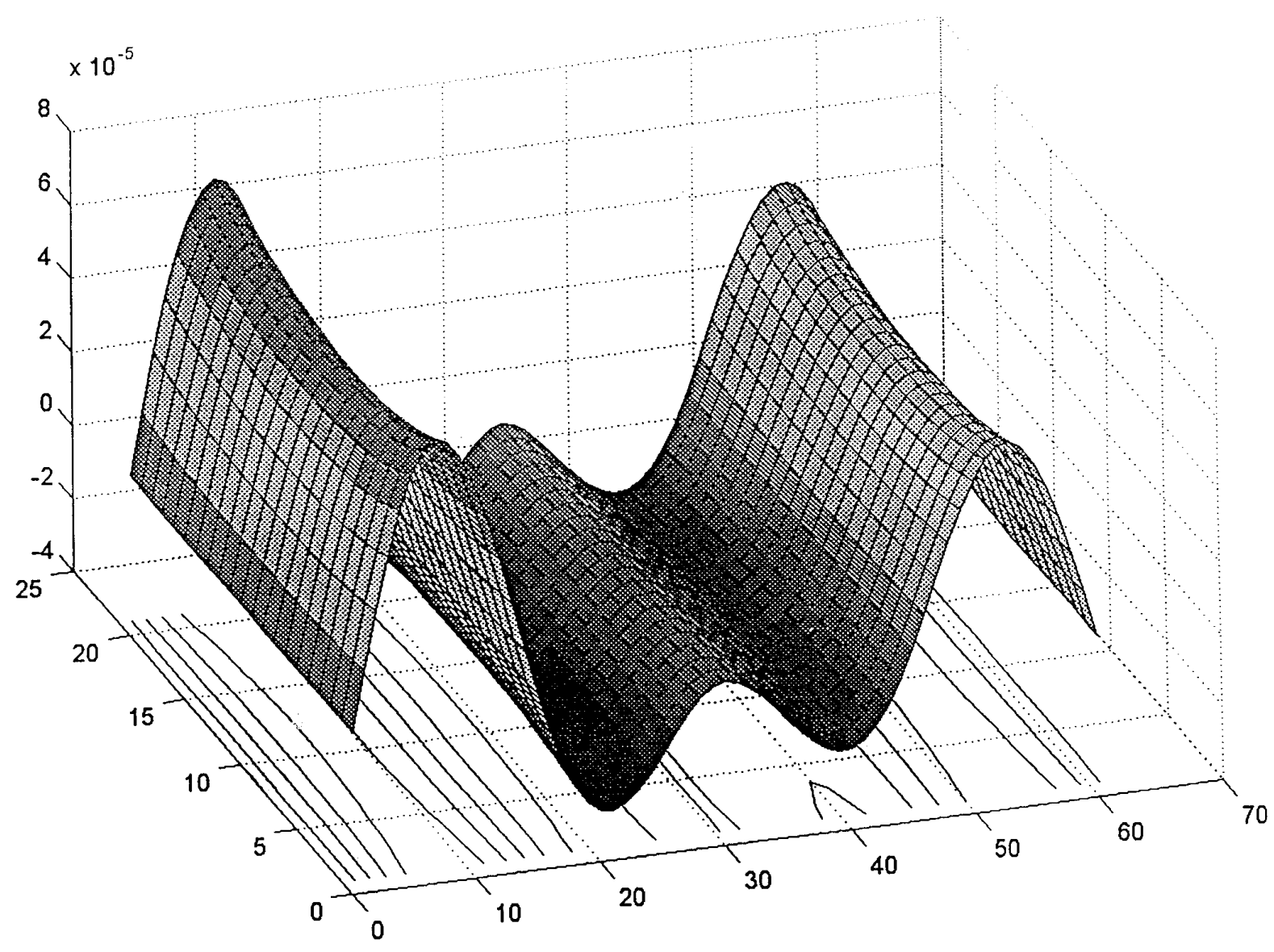

Fig. 10. Second mode shape of 4-span deck, $\phi_{1}=\phi_{3}=\phi_{4}=1.0, \phi_{2}=1.1$.

Table 3

Computed 4-Span Deck Eigenvalues, $\lambda_{b}^{2}=\omega b^{2} \sqrt{ } \rho / D, \phi_{1}=\phi_{3}=\phi_{4}=1.0$. Comparison values obtained from Multi-Span Approach, in brackets (* indicates classical values)

\begin{tabular}{cllllllll}
\hline Mode & \multicolumn{7}{c}{$\phi_{2}$} \\
\cline { 2 - 8 } & \multicolumn{2}{c}{1.0} & \multicolumn{2}{c}{1.05} & \multicolumn{2}{c}{1.15} & \multicolumn{2}{c}{1.25} \\
\hline 1 & $9.568^{*}$ & $(9.568)$ & 9.778 & $(9.778)$ & 10.05 & $(10.05)$ & 10.22 & $(10.22)$ \\
2 & 11.22 & $(11.22)$ & 11.34 & $(11.34)$ & 11.55 & $(11.55)$ & 11.74 & $(11.74)$ \\
3 & $15.13^{*}$ & $(15.13)$ & 15.48 & $(15.48)$ & 15.99 & $(16.00)$ & 16.33 & $(16.33)$ \\
4 & 15.88 & $(15.88)$ & 16.10 & $(16.10)$ & 16.34 & $(16.34)$ & 16.45 & $(16.45)$ \\
5 & 17.13 & $(17.14)$ & 17.24 & $(17.25)$ & 17.44 & $(17.44)$ & 17.59 & $(17.60)$ \\
6 & 20.34 & $(20.35)$ & 20.69 & $(20.71)$ & 21.16 & $(21.18)$ & 21.42 & $(21.44)$ \\
\hline
\end{tabular}

more column and one more row of matrix segments.

It is not practical to try and provide mode shape information associated with all of the above eigenvalues. Nevertheless, it is instructive to examine a very limited number of these mode shapes.

In Figs 6 through 8, three dimensional plots for the first, second, and third modes, respectively, of free vibration of a 3-span deck with $\phi_{1}=\phi_{3}=1.0$, and $\phi_{2}=1.1$, are presented. It will be appreciated that for such a deck configuration, not only will all modes be symmetric, or anti-symmetric, about the long central axis of the deck, but each mode will be symmetric or anti-symmetric about the short central axis as well.

The first free vibration mode is depicted in Fig. 6. It is seen to be symmetric about each axis as discussed above. In this Figure, as in all mode shape Figures, we observe anticlastic bending due to the Poisson effect. It is clear in Fig. 6, for example, that a line drawn along the short central axis toward the free edge, curves outward as it approaches the edge. This phenomenon 


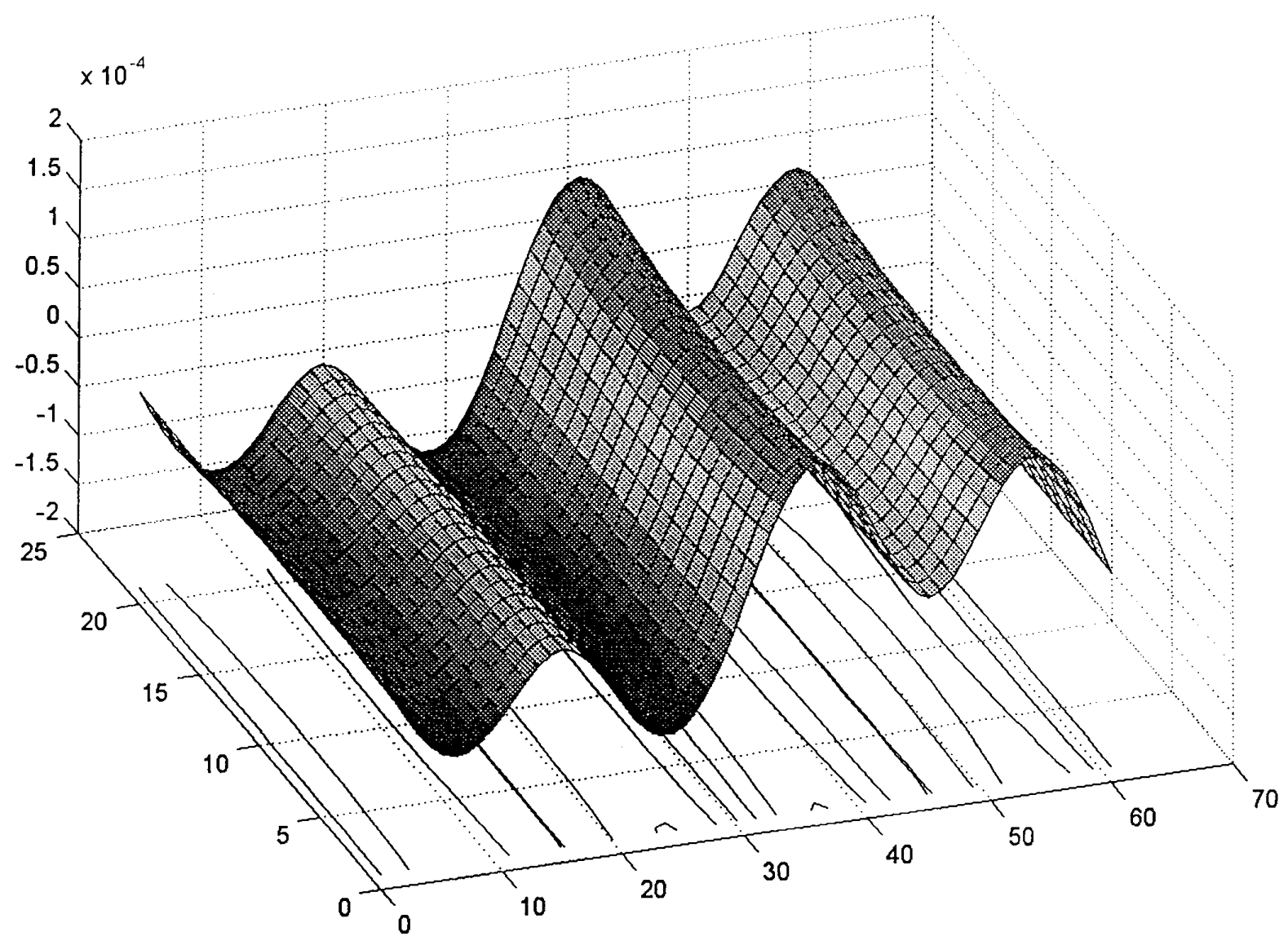

Fig. 11. Third mode shape of 4-span deck, $\phi_{1}=\phi_{3}=\phi_{4}=1.0, \phi_{2}=1.1$.

may be of interest to people investigating wind induced vibration of bridge decks.

The second mode of free vibration as shown in Fig. 7, is seen to be anti-symmetric about the short central axis, while it is symmetric about the long central axis.

The third free vibration mode, while symmetric about the short central axis, is the first mode found to be anti-symmetric with respect to the long central axis. Such modes would be of particular interest to researchers concerned with bridge excitation resulting from winds blowing across the deck.

Mode shapes associated with first, second, third and fourth mode vibration of a 4-span deck are depicted in Figs 9 through 12. It has been arbitrarily chosen to examine a deck with aspect ratios $\phi_{1}, \phi_{3}$ and $\phi_{4}$ equal to 1.0. Aspect ratio $\phi_{2}$ is set equal to 1.1 .

The first three modes are all symmetric with respect to the long central axis. It is only in the fourth mode that we encounter anti-symmetry about this axis. Again, this phenomenon will be of interest to people studying the effects of cross-winds on bridge behavior.
One should recall that with the lateral displacement (mode shape) associated with any natural frequency established, distributions of dimensionless bending moments, transverse shear forces, and twisting moments are available throughout the entire bridge deck. One need only utilize standard formulae available in most texts dealing with rectangular plate behaviour, which express these moments, shear forces, etc., in terms of the plate lateral displacement and material properties.

\section{Conclusions}

The goal of developing an efficient analytical approach for predicting accurate free vibration eigenvalues and mode shapes of multi-span bridge decks has been achieved. Convergence is found to be rapid and the addition of extra spans to the deck is very easily taken care of in the analysis. It is natural when investigating the free vibration of a problem of the type discussed here to consider analyzing each span sepa- 


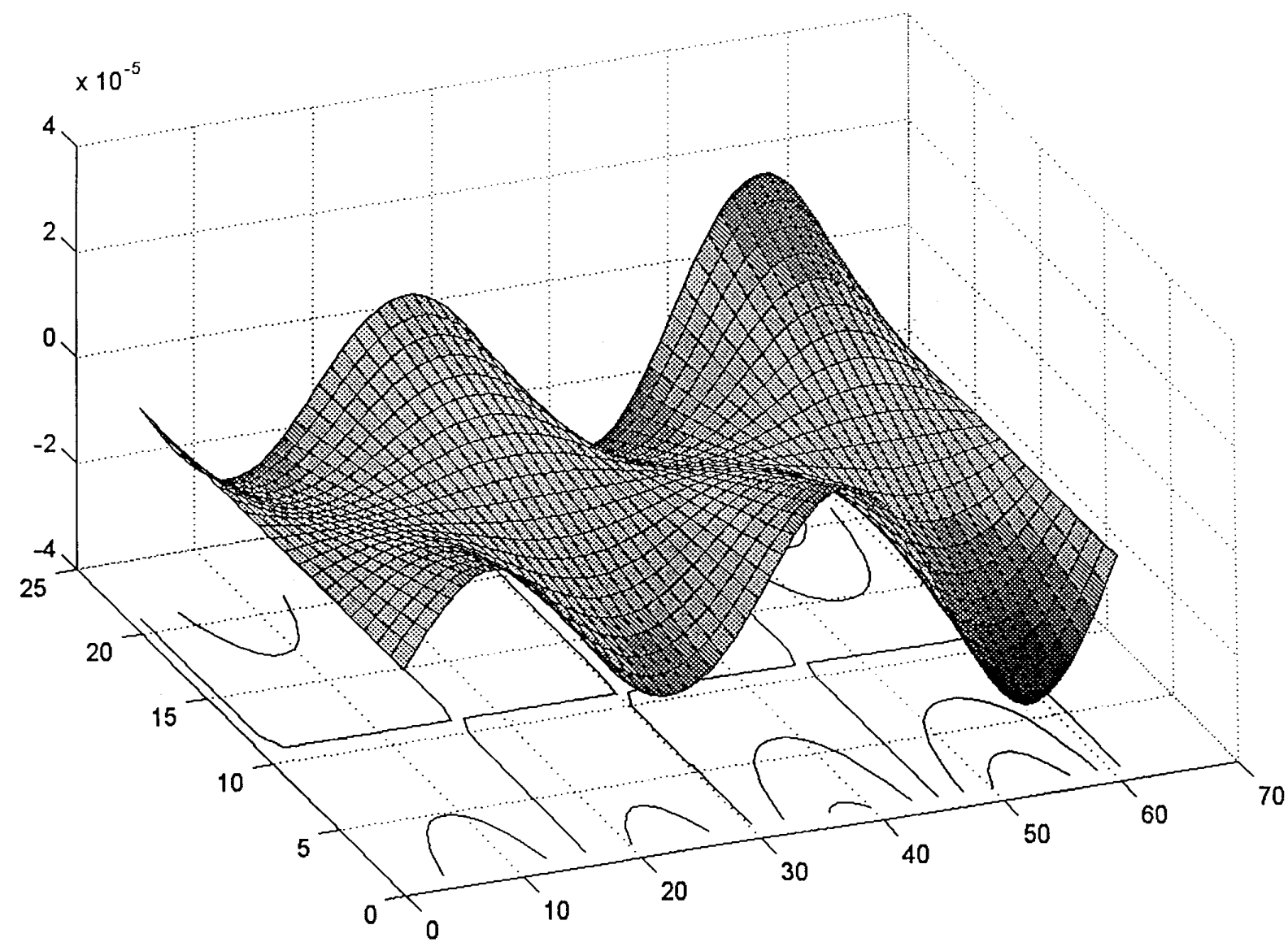

Fig. 12. Fourth mode shape of 4-span deck, $\phi_{1}=\phi_{3}=\phi_{4}=1.0, \phi_{2}=1.1$.

rately and then enforce continuity at the various span interfaces. In fact, that is what was done in carrying out the auxiliary 'multi-span approach' referred to earlier. The efficiency of the method described here lies in the fact that only one rectangular plate, the overall bridge deck, need be analyzed. Two steps are involved. One is to ensure that the long running edges are free of vertical edge reaction and bending moment. The other step is to ensure that lateral displacement of the above continuous plate is forbidden at inter-span locations. It was also seen that with the present approach only one column and one row need be added to the basic eigenvalue matrix for each additional span incorporated into the bridge deck.

Another advantage of the present analytical approach is that, unlike the Rayleigh-Ritz approach, for example, no functions need be selected to represent bridge deck deflection. Also, the governing differential equation is satisfied exactly throughout the entire domain of the structure.
A fairly simple computer routine is currently capable of handling decks of any number of spans. The reader will appreciate that with addition of further spans the number of terms utilized in the building block solution series must be increased. This will be necessary to ensure that net residual bending moment along the free edges of the deck will be made sufficiently small.

It is recognized that the present theoretical model handles decks of the most simple design, i.e., the deck is treated as a thin isotropic rectangular plate. It is known, however, that the superposition method as exploited here has been shown to be capable of handling orthotropic as well as thick plate problems $[3,4]$. There is no doubt that the present theoretical approach can be extended to handle bridge decks with the above and other complicating factors.

It will be appreciated that the computational procedure described here can be readily employed to decide upon optimum locations for bridge deck inter-span supports when such discression is available to the designer. 
One may, for example, wish to arrange support distributions in such a way as to maximize the fundamental frequency of the deck. Another possibility is that one may wish to 'tune-out' a particular frequency in order to avoid a resonant condition.

\section{Nomenclature}

a Length of bridge deck

$a_{1}, a_{2} \quad$ Length of first and second spans

$b \quad$ Width of deck

$D \quad$ Flexural rigidity of deck

$K$ Number of terms utilized in series solutions

$M$ Bending moment

$M b^{2} / a D$ Dimensionless bending moment associated with $\eta$ direction

$P \quad$ Amplitude of line loading per unit length

$P^{*}=-P b^{3} / a D$

$u$ Distance to line loading divided by ' $a$ '

$u_{1}, u_{2}$ Dimensionless distances to first and second inter-span supports, respectively.

$\nu \quad$ Distance to line loading divided by ' $b$ '

$W$ Deck lateral displacement divided by ' $a$ ' $\phi \quad$ Deck aspect ratio, $b / a$

$\phi_{1}, \phi_{2}$ Aspect ratios of first and second spans, equals $b / a_{1}$, and $b / a_{2}$, respectively

$$
\begin{aligned}
& \lambda^{2}=\omega a^{2} \sqrt{ } \rho / D \\
& \lambda_{b}^{2}=\omega b^{2} \sqrt{ } \rho / D \\
& \omega \quad \text { Circular frequency of vibration } \\
& \rho \quad \text { Mass of deck per unit area } \\
& \nu \quad \text { Poisson ratio of deck material } \\
& \nu^{*}=2-\nu \\
& \xi \quad \text { Coordinate distance divided by ' } a \\
& \eta \quad \text { Coordinate distance divided by ' } b
\end{aligned}
$$

\section{References}

[1] D.J. Gorman, World Scientific Publishing Co. Vibration Analysis of Plates by The Superposition Method, 1999.

[2] D.J. Gorman, Elsevier North Holland Co. Free Vibration Analysis of Rectangular Plates, 1982.

[3] D.J. Gorman, Free Vibration Analysis of Clamped Orthotropic Plates by The Superposition Method, Jour. of Sound and Vibration 140(31) (1990), 391-411.

[4] D.J. Gorman and W. Ding, Accurate Free Vibration Analysis of The Completely Free Rectangular Mindlin Plate, Jour. of Sound and Vibration 189(3) (1996), 341-353. 

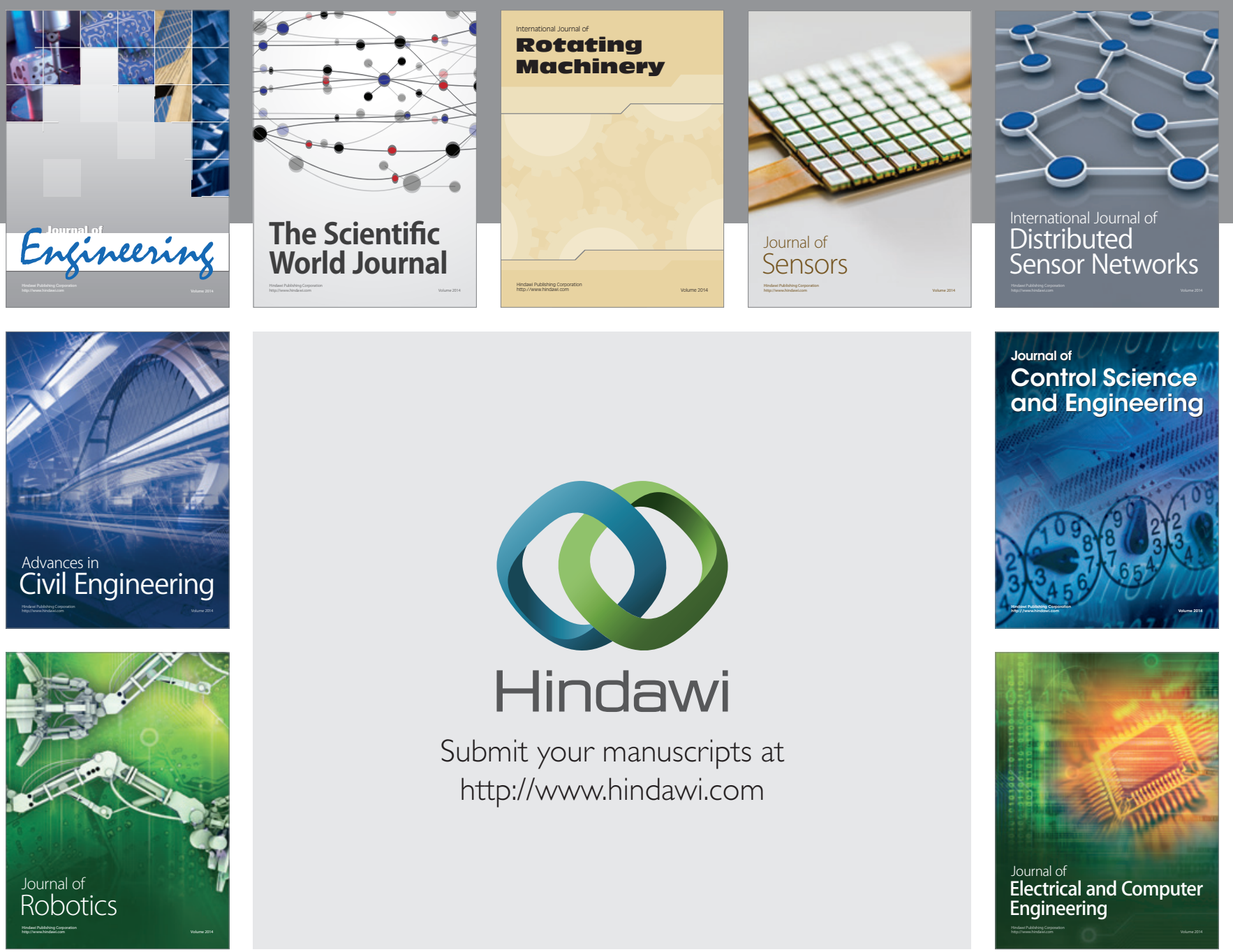

Submit your manuscripts at

http://www.hindawi.com
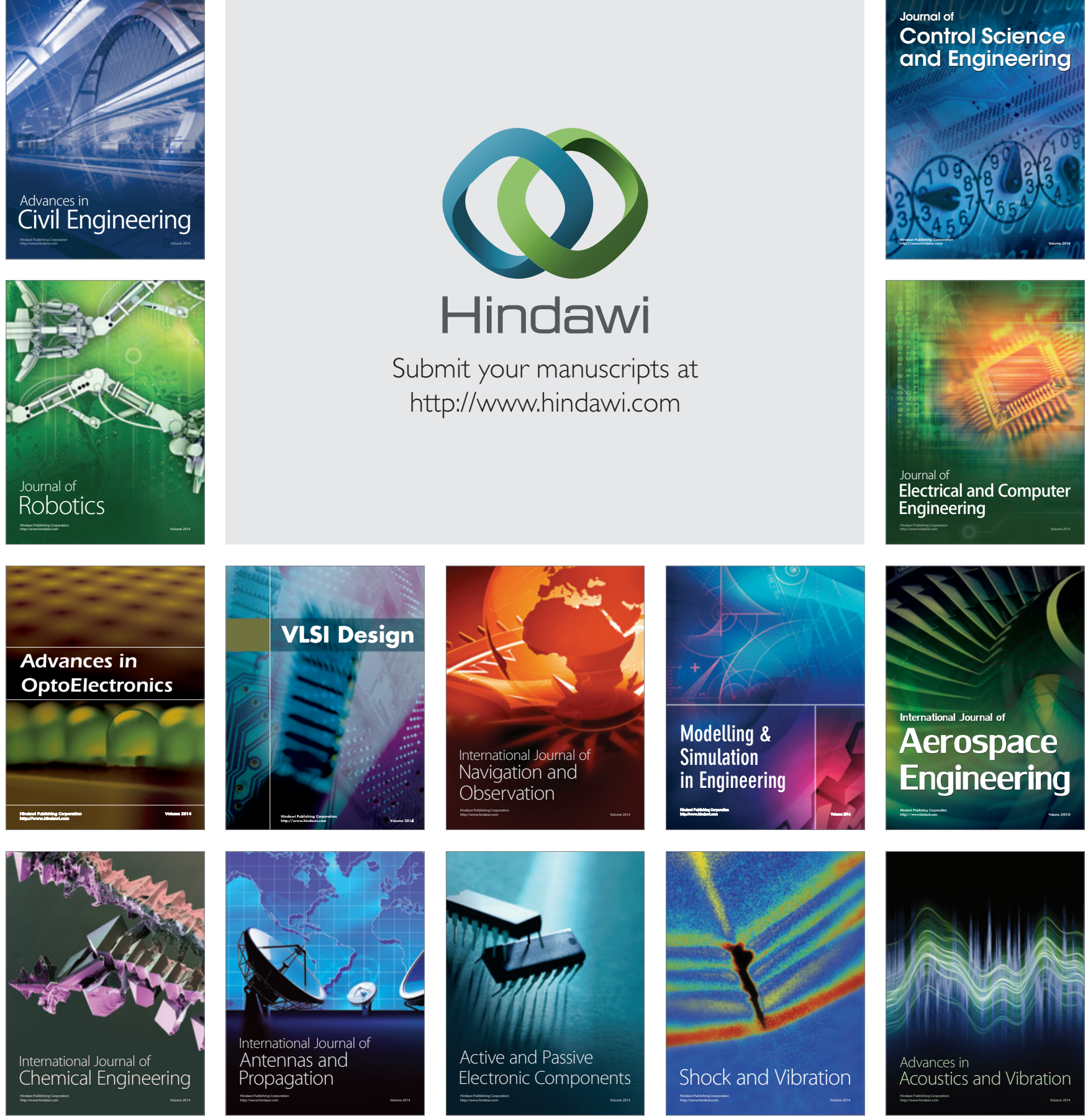